\begin{tabular}{|l|c|c|c|}
\hline & SLE & CTD non-SLE & Chronic arthritis \\
\hline Sterility & $3(12 \%)$ & 0 & $2(7.6 \%)$ \\
\hline Miscarriages & $8(32 \%)$ & 0 & $7(26 \%)$ \\
\hline Assisted reproduction & 3 IVF (12\%) & $1(11 \%)$ & 2 insemination (7.6\%) \\
\hline Preterm delivery & 2 insemination (4\%) & 0 & 0 \\
\hline Flares during pregnancy & 0 & 0 & $11(42 \%)$ \\
\hline Preeclampsia & $2(8 \%)$ & 0 & 0 \\
\hline
\end{tabular}

IVF: in vitro fecundation

chronic arthritis (CA) including rheumatoid arthritis (RA), spondyloarthritis (SPA) and psoriatic arthritis (PsA).

Results 60 women (25 SLE, 9 connective tissue diseases nonSLE, 26 CA) were evaluated for pregnancy planning. All of them were in remission and were taking safe pharmacological treatment for the pregnancy. Fifty percent presented advanced maternal age ( $>35$ years old) at the time of evaluation (8 SLE, 2 CTD no SLE and 15 CA).

Until now, 48 patients have finished their pregnancy (18 SLE, 8 CTD non-SLE and $22 \mathrm{CA}$ ); in the others pregnancy is still on going.

Data collected is showed in table.

Sterility and fertility were similar in both, SLE and CA, and comparable with healthy women of the same age. Preeclampsia and an increased risk of preterm delivery were more frequent in the outcome of patients with SLE ( $p>0.05)$, however patients with CA showed basically joint inflammation $(p>0.05)$. Antiphospholipid antibodies were not associated with higher rates of either fetal or maternal complications in this group of patient, probably because treatment with aspirin and LMWH was started at the beginning of conception. All neonates whose mothers were anti Ro positive (16 women) had positive anti Ro serum determination, however only four of them presented with atrioventricular block (AVB).

Conclusion in our group of patients, SLE showed comparable results of fertility with healthy women of the same age, probably because disease was in completed remission and they never use cyclophosphamide. Pregnancy planning help ensure better outcomes in these patients

\section{PS4:86 OBSTETRIC AND NEONATAL OUTCOMES IN SYSTEMIC LUPUS ERYTHEMATOSUS: A POPULATION-BASED REGISTER STUDY}

${ }^{1} \mathrm{LS}$ Andersen, ${ }^{3} \mathrm{M}$ Bliddal, ${ }^{2} \mathrm{LL}$ Andersen, ${ }^{1} \mathrm{~A}$ Voss. ${ }^{1}$ Department of Clinical Research, Research Unit of Rheumatology, University of Southern Denmark, Odense, Denmark; ${ }^{2}$ Department of Gynaecology and obstetrics, Odense University Hospital, Odense, Denmark; ${ }^{3}$ OPEN - Odense Patient data Explorative Network, University of Southern Denmark, Odense, Denmark

\subsection{6/lupus-2018-abstract.131}

Introduction In women with systemic lupus erythematosus (SLE) pregnancies may be negatively affected by disease activity and medical treatment. Increased frequencies of adverse outcome including preterm delivery and perinatal morbidity/ mortality have been reported. However, different frequencies are reported from different studies.
Aim and hypothesis Utilising national health registers we want to investigate maternal and fetal outcomes in Danish pregnant SLE patients compared to outcomes in the background population.

Methods The outcome of pregnancies in Danish SLE patients in 1997-2016 is studied utilising healthcare-registries (the Danish National Patient Registry (NPR) and the Medical Birth Register (MBR)) and trends are described.

Study population and material: All females with a diagnosis of SLE in the study period are identified from the NPR (ICD-8 and ICD-10 diagnoses of SLE). The outcome of all pregnancies (routinely registered in NPR and MBR) is compared to the outcome in an age-matched cohort of pregnant women without SLE (each SLE patient is matched with 20 females from the background population). Data about infants are retrieved from the MBR

Statistics: Using logistic regression we will examine if risk of adverse maternal and fetal outcome is higher in women with SLE than in women without SLE, by calculating crude and adjusted odds ratios

Results Frequencies of adverse maternal outcomes including preeclampsia, preterm delivery and Caesarean section, and adverse infant outcome including asphyxia, growth retardation and low Apgar score will be presented. Crude and adjusted odds ratios will be calculated comparing pregnancies in SLE females with pregnancies of non-SLE females.

Data are at present being retrieved from the Danish authorities and subsequent data analysis is expected to be completed December 2017.

Approximately 800 women with SLE are expected to be included in the study.

\section{Poster session 5: Innate and adaptive immunity}

\section{PS5:87 JUVENILE-ONSET SLE IMMUNOPATHOGENESIS COULD BE ASSOCIATED WITH ALTERED IMMUNE CELL PLASMA MEMBRANE LIPIDS AND LIPOPROTEIN METABOLISM}

${ }^{1} \mathrm{G}$ Robinson, ${ }^{2}$ I Pineda-Torra, ${ }^{1} Y$ loannou, ${ }^{1} \mathrm{E}$ Jury. ${ }^{1}$ University College London Rheumatology and Adolescent Rheumatology, London, UK; ${ }^{2}$ University College London Clinical Pharmacology, London, UK

10.1136/lupus-2018-abstract.132 
Background Juvenile-onset systemic lupus erythematosus (JSLE) is an autoimmune disorder characterised by immune dysregulation, chronic inflammation and increased cardiovascular risk. Our findings in adult-onset SLE link immune cell dysregulation with defects in plasma membrane signalling platforms (lipid rafts). In JSLE little is known about the immune profile or whether abnormal lipid metabolism contributes to pathogenesis.

Methods Flow cytometry was used to measure metabolic marker expression on immune cell subsets from 39 healthy donors (HCs) and 35 JSLE patients. Metabolic biomarker analysis including lipoprotein composition was performed on matching serum.

Results JSLE patients had significantly elevated membrane lipid rafts in T-cells, B-cells and plasmacytoid dendritic cells compared to HCs suggesting dysregulated membrane receptor signalling. Furthermore, lipid raft expression correlated positively with cell activation markers, disease activity, erythrocyte sedimentation rate and dsDNA titre and negatively with complement protein C3 supporting the hypothesis that altered metabolism is associated with JSLE pathogenesis. Importantly, ROC curve analysis showed that lipid raft expression on these cell types is an excellent diagnostic of high disease activity in JSLE. Metabolomic analysis of matching serum revealed that high disease activity patients had significantly decreased atheroprotective high density lipoproteins (HDL) and increased atherogenic low density lipoproteins (LDL) suggesting altered transport of lipids. In addition, lipids associated with membrane rafts such as sphingomyelin, phosphatidylcholine, phosphoglycerides and cholesterol correlated negatively with HDL in high disease activity patients but positively in low disease activity patients. Immune cell lipid rafts correlated positively with LDL and negatively with HDL together suggesting altered lipid uptake/efflux from these cells; this may alter immune cell signalling in JSLE patients. Stratification of patients based on their lipid profile by hierarchical clustering revealed 3 groups that were unique in both immnophenotype and clinical presentation.

Conclusions Differences in the metabolic profiles of immune cell subsets and lipoprotein lipid transport in JSLE contribute to disease pathogenesis and severity. Regulation of lipid metabolism may therefore have therapeutic benefit for JSLE patients providing a dual effect of reducing inflammation and atherosclerotic risk. These therapeutics may perform better in patients that present specific clinical and phenotypic features.

\section{PS5:88 ALTERED CELL SURFACE N-GLYCOSYLATION OF AUTOIMMUNE T-CELLS RESULTS IN DECREASED BINDING OF IMMUNOREGULATORY GALECTIN-1}

'L Kovács, ${ }^{2} E$ Szabó, ${ }^{1,2} A$ H Hornung, ${ }^{2} E ́$ Monostori, ${ }^{2} A$ Czibula. ${ }^{1}$ University of Szeged, Faculty of Medicine, Dept of Rheumatology and Immunology, Szeged, Hungary; ${ }^{2}$ Biological Research Centre of the Hungarian Academy of Sciences, Dept of Genetics, Lymphocyte Signal Transduction Lab, Szeged, Hungary

10.1136/lupus-2018-abstract.133

Glycosylation (sugar code) is frequently altered on the surface of various cells in patients with immune-mediated diseases resulting in changes in signal transduction and metabolic control. However, alteration of glycosylation patterns of $\mathrm{T}$ cells has not yet been characterised in autoimmune disorders. We have previously demonstrated that T-cells of patients with systemic lupus erythematosus (SLE) are resistant to the apoptotic effects of galectin-1 (Gal-1), an immunoregulatory lectin.

T-cells from patients with active SLE $(n=18)$, rheumatoid arthritis (RA) $(n=14)$ or Sjögren's syndrome (SS) $(n=14)$ and from healthy controls $(n=19)$ were examined. Cell surface glycosylation was analysed with lectin-binding assay, and the expression of glycosyltransferase and glycosidase enzymes participating in the construction of the glycan chains was measured with reverse transcriptase polymerase chain reaction (RTPCR).

Resting SLE T-cells bound significantly higher amounts of several lectins, while RA and SS resting T-cells were similar to controls in their glycosylation pattern. Activated T-cells from all autoimmune groups bound significantly less Gal-1 than controls, while other lectins bound similarly. Gene expression including alpha mannosidases (MAN1A1, MAN1A2, MAN2A1 and MAN2A2) and beta-N-acetylglucosaminyltransferases (MGAT1-5) in autoimmune activated T-cells did not differ from controls with the only exception of MAN1A2 in SS. However, we found a significant increase in the mRNA ratios of certain sialyltransferases and neuraminidases, specifically in ST6GAL1/NEU1 in SLE and SS, and in ST3GAL6/NEU1 in SLE and RA patients compared to controls. Treatment of cells with neuraminidase resulted in a remarkable increase in Gal-1 binding

The glycosylation pattern of resting SLE T-cells was consistent with a pre-activated phenotype. Decreased Gal-1 binding found in all three diseases can be explained with an increased terminal sialylation, which may be a consequence of an altered expression of sialyltransferase and neuraminidase genes, whose concerted action is responsible for the degree of sialylation of glycan structures. Accordingly, neuraminidase treatment resulted in remarkably increased Gal-1 binding. We propose that increased sialylation may at least partially explain the previously found resistance to the immunoregulatory effects of Gal-1 in SLE, hence contributing to the pathomechanism of the examined diseases.

\section{PS5:89 LOOKING FOR A SLE SIGNATURE ON PERIPHERAL B CELL SUBSETS: DOES A PREPONDERANT CD38 POSITIVE PLASMABLAST-SUBPOPULATION LACK CD73 AS A SIGN OF A DIMINISHED B REGULATORY POOL?}

M Siekierka-Harreis, M Schroedter, G Chehab, J Richter, S Vordenbäumen, M Schneider, G Pongratz. Rheumatology, Medical Faculty Heinrich-Heine University, Düsseldorf, Germany

\subsection{6/lupus-2018-abstract.134}

Background Systemic Lupus Erythematosus (SLE) is an autoimmune disorder characterised by polyclonal Bcell activation, production of dsDNA-autoantibodies and cytokines. Subsets of Bcells play a central role in SLE-pathogenesis. The inflammatory milieu is characterised by the accumulation of adenosine, which confers immunosuppressive effects. In SLE, the role of CD73, an enzyme involved in the extracellular generation of adenosine from ATP, is not well characterised. This study aimed to characterise expression of CD73 B cell subsets of SLE-patients as compared to healthy controls (HC).

Methods B cell subsets were characterised from peripheral blood of 23 SLE patients attending the outpatient clinic at the Rheumatology Unit of University Hospital Düsseldorf and of $15 \mathrm{HC}$ by FACS. All patients fulfilled the revised SLE-criteria 